\title{
Latest development of a W-band Gyro-TWA based on a Helically Corrugated Interaction Region
}

\author{
W. He, C. R. Donaldson, L. Zhang, P. McElhinney, K. Ronald, \\ A. W. Cross and A. D. R. Phelps \\ Department of Physics, SUPA, \\ University of Strathclyde, Glasgow, G4 ONG, UK \\ Email: w.he@strath.ac.uk
}

\begin{abstract}
Latest development and experimental study of a $W$-band gyro-TWA with a helically corrugated waveguide and a cusp electron gun are presented. With an input seed signal from an $1.5 \mathrm{~W}, 90-96 \mathrm{GHz}$ solid state source a gain of $27 d B$ was measured from the experiment. The bandwidth of the gyro-TWA was measured to be at least 5 $\mathrm{GHz}$.
\end{abstract}

Keywords: helically corrugated waveguide, gyrotron, gyro-TWA, W-band gyro-devices, cusp electron gun.

\section{Introduction}

The gyro-devices are high power coherent microwave sources that excel at high frequencies (up to the terahertz range). There are a number of applications for such devices including high resolution RADAR, plasma diagnostics, communications and medical imaging, and accurate material analysis based on electron spin resonance in conjunction with magnetic resonance and terahertz spectroscopy. Recently gyro-devices in the form of both a gyro-TWA [1] and a gyro-BWO [2,3] have been developed at the University of Strathclyde. In this paper the latest development and experimental results of a W-band gyroTWA are presented. A CAD drawing of the gyro-TWA including its components such as input coupler, output window and magnetic coils is shown in Fig. 1.

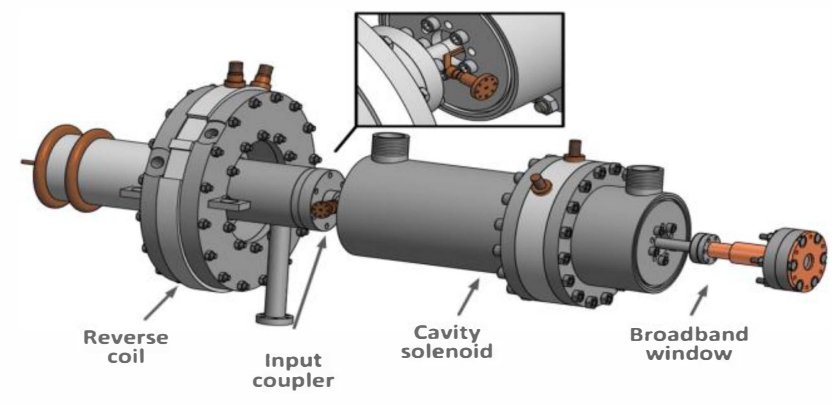

Figure 1. A CAD drawing of the W-band gyro-TWA.

\section{Interaction region}

When the interaction region has a helical corrugation on the inner surface there exists an "ideal" eigenwave giving many benefits that can be exploited in novel gyro-devices and in pulse compression [4]. For the gyro-devices the eigenwave has an almost constant value of group velocity over a wide frequency band in the region of small axial wave numbers [5]. This dispersion can be designed to match the dispersion line of an electron cyclotron mode or its harmonics. This allows broadband microwave amplification to be achieved in a gyrotron travelling wave amplifier [6].

To drive the beam-wave interaction in a helically corrugated interaction region (HRIC) it is necessary to use an axis-encircling electron beam. The HCIR resonantly couples the $\mathrm{TE}_{11}$ and $\mathrm{TE}_{21}$ modes and results in a ' $\mathrm{TE}_{21}$ ' like' eigenmode. This eigenmode therefore can efficiently interact with the second harmonic of the electron cyclotron frequency. An axis-encircling electron beam is ideal for harmonic operation of gyro-devices as the mode selectivity nature of such a beam requires that the harmonic number is equal to the azimuthal index of a waveguide mode for effective beam wave coupling, which leads to a reduced possibility of parasitic oscillations. Such an axis-encircling electron beam can be generated by a cusp [7]. The maximum output of the gyro-TWA was predicted to be 5 $\mathrm{kW}$ with a $3 \mathrm{~dB}$ frequency bandwidth of $90-100 \mathrm{GHz}$ and a saturated gain of $37 \mathrm{~dB}$.

The coupling of the beam and the eigenwave in the gyroTWA that exists inside the helically corrugated waveguide can be described by the following equation [5]:

$$
\begin{aligned}
& {\left[\left(h^{2}-2 \delta\right)\left(h+\Delta_{g}-\delta / h_{\bullet}\right)+2 \sigma^{2} / h_{\bullet}\right]\left[h-\left(\delta-\Delta_{H}\right) / \beta_{z \bullet}\right]^{2}} \\
& =C^{3}\left(h+\Delta_{g}-\delta / h_{\bullet}\right)\left\{1+\frac{2 s}{\alpha_{\bullet}^{2} \beta_{z \bullet}}\left[h-\left(\delta-\Delta_{H}\right) / \beta_{z \bullet}\right]\right\}
\end{aligned}
$$

\section{Cusp electron gun}

The cusp electron gun has been simulated and experimentally measured [8] to show the generation of a $1.5 \mathrm{~A}, 40 \mathrm{keV}$ axis-encircling electron beam. The alpha value has been measured over a range from 1.0 to 3.0. The results agree well with the simulations.

The reverse field coil is located behind the cathode and the main cavity coil is in front. When the magnetic field is varied between $1.65 \mathrm{~T}$ and $2.1 \mathrm{~T}$ the reverse coil's magnetic field can be adjusted to keep a constant beam pitch angle.

\section{Input and output of the wave}

The input signal was inserted using an input coupler, which operated from $84 \mathrm{GHz}$ to $104 \mathrm{GHz}$. It consists of a pillbox window with a brazed ceramic disk to seal the UHV vacuum. The coupler was improved from previous version and optimized to allow for mechanical robustness and 
larger machining tolerances. To allow maximum beam transportation in the coupler region, the beam tunnel incorporated a Bragg reflector at the diode side of the coupler so that input wave would propagate downstream into the interaction region. The input coupler achieved -10 $\mathrm{dB}$ reflection in the frequency band.

The improved output window consisted of 3 disks, with the middle ceramic disk serving as the vacuum boundary and two quartz disks on each side [9]. It achieved a low reflection of $-30 \mathrm{~dB}$ or better in the $90-100 \mathrm{GHz}$ frequency band.

\section{Experiment}

The gyro-TWA experiments proved successful. A photograph of the device is shown in Fig. 2. Many components have been measured including: broadband input coupler, corrugated quasi-optical mode converter, dispersion of the helical interaction region and broadband microwave window. The output microwave radiation was detected by two crystal detectors situated inside screened boxes. The output power was calibrated using a known microwave source. The experimental results including the output powers and operating frequency bands are measured. With an input seed signal from an 1.5 W, 90-96 $\mathrm{GHz}$ solid state source a gain of $27 \mathrm{~dB}$ was measured from the experiment. The bandwidth was measured to be at least $5 \mathrm{GHz}$.

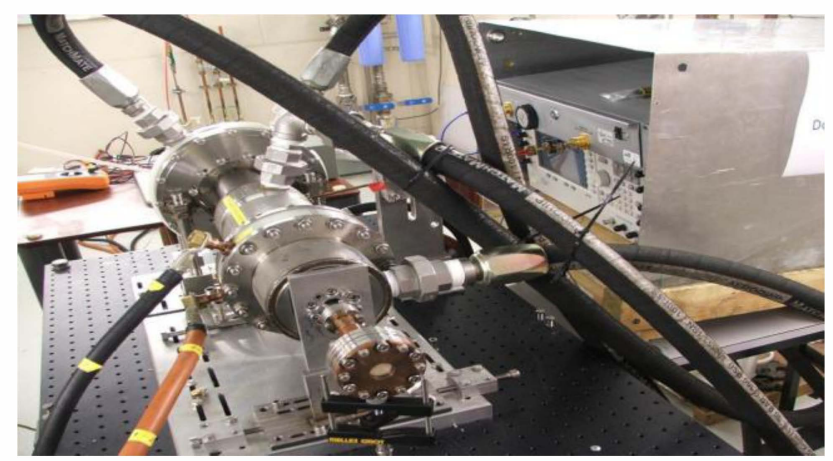

Figure 2. A photograph of the W-band gyro-TWA experiment.

\section{Conclusion}

Theory and numerical simulations of the operating parameters of helical waveguide W-band gyro-devices have been confirmed by the experimental measurements.

\section{Acknowledgements}

The authors would like to thank EPSRC UK (research grant EP/G036659/1) for supporting this work and Dr. P. Huggard, Mr M. Beardsley and Mr. P. Hiscock of the Millimetre Wave Technology Group at the STFC
Rutherford Appleton Laboratory, UK for the construction of the HCIR.

\section{References}

[1] A. W. Cross, W. He, A. D. R. Phelps, K. Ronald, C. G. Whyte, A. R. Young, C. W. Robertson, E. G. Rafferty, and J. Thomson, "Helically corrugated waveguide gyrotron traveling wave amplifier using a thermionic cathode electron gun," Appl. Phys. Lett., 90, (25), 253501, 2007.

[2] W. He, A. W. Cross, A. D. R. Phelps, K. Ronald, A. R. Young, C. G. Whyte, E. G. Rafferty, C. W. Robertson, J. Thomson, S. V. Samsonov, V. L. Bratman, and G. G. Denisov, "Theory and simulations of a gyrotron backward wave oscillator using a helical interaction waveguide", Appl. Phys. Lett., 89, (9), 091504, 2006.

[3] W. He, C.R. Donaldson, L. Zhang, K. Ronald, et al., "High power wideband gyrotron backward wave oscillator operating towards the terahertz region," Phys. Rev. Lett. 110, 165101, 2013.

[4 S. V. Samsonov, A. D. R. Phelps, V. L. Bratman, G. Burt, G. G. Denisov, A. W. Cross, K. Ronald, W. He, and H. Yin, "Compression of frequency-modulated pulses using helically corrugated waveguides and its potential for generating multigigawatt rf radiation," Phys. Rev. Lett., 92, (11), 118301, 2004.

[5] L. Zhang, W. He, K. Ronald, A. D. R. Phelps, C. G. Whyte, C. W. Robertson, A. R. Young, C. R. Donaldson, and A. W. Cross, "Multi-Mode Coupling Wave Theory for Helically Corrugated Waveguide," IEEE Trans. Microwave Theory Tech., 60, (1), pp. 1-7, 2012.

[6] G. G. Denisov, V. L. Bratman, A. W. Cross, W. He, A. D. R. Phelps, K. Ronald, S. V. Samsonov, and C. G. Whyte, "Gyrotron traveling wave amplifier with a helical interaction waveguide," Phys. Rev. Lett., 81, (25), pp. 5680-5683, 1998.

[7] W. He, C. G. Whyte, E. G. Rafferty, A. W. Cross, A. D. R. Phelps, K. Ronald, A. R. Young, C. W. Robertson, D. C. Speirs, and D. H. Rowlands, "Axis-encircling electron beam generation using a smooth magnetic cusp for gyrodevices," Appl. Phys. Lett., 93, (12), 121501, 2008.

[8] C. R. Donaldson, W. He, A. W. Cross, F. Li, A. D. R. Phelps, L. Zhang, K. Ronald, C. W. Robertson, C. G. Whyte, and A. R. Young, "A cusp electron gun for millimeter wave gyro-devices," Appl. Phys. Lett., 96, (14), $141501,2010$.

[9] C. R. Donaldson, W. He, L. Zhang, and A. W. Cross, "A W-band multi-layer microwave window for pulsed operation of gyro-devices," IEEE Microw. Wireless Compon. Lett., vol. 23, pp. 237-239, 2013. 